\title{
Intronic and plasmid-derived regions contribute to the large mitochondrial genome sizes of Agaricomycetes
}

\author{
Kajsa Himmelstrand • Åke Olson • \\ Mikael Brandström Durling • Magnus Karlsson • \\ Jan Stenlid
}

Received: 22 November 2013 / Revised: 30 May 2014 / Accepted: 21 June 2014 / Published online: 11 July 2014

(C) The Author(s) 2014. This article is published with open access at Springerlink.com

\begin{abstract}
Sizes of mitochondrial genomes vary extensively between fungal species although they typically contain a conserved set of core genes. We have characterised the mitochondrial genome of the conifer root rot pathogen Heterobasidion irregulare and compared the size, gene content and structure of 20 Basidiomycete mitochondrial genomes. The mitochondrial genome of $H$. irregulare was $114,193 \mathrm{bp}$ and contained a core set of 15 protein coding genes, two rRNA genes and 26 tRNA genes. In addition, we found six non-conserved open reading frames (ORFs) and four putative plasmid genes clustered in three separate regions together with 24 introns and 14 intronic homing endonuclease genes, unequally spread across seven of the core genes. The size differences among the 20 Basidiomycetes can largely be explained by length variation of intergenic regions and introns. The Agaricomycetes contained the nine largest mitochondrial genomes in the Basidiomycete group and Agaricomycete genomes are significantly $(p<0.001)$ larger than the other Basidiomycetes. A feature of the Agaricomycete mitochondrial genomes in this study was the simultaneous occurrence of putative plasmid genes and non-conserved ORFs, with Cantharellus cibarius as only exception, where no non-conserved ORF was identified. This indicates a mitochondrial plasmid origin of the non-conserved ORFs or increased mitochondrial genome dynamics of species harbouring mitochondrial plasmids. We hypothesise that two independent factors are the driving
\end{abstract}

Communicated by S. Hohmann.

K. Himmelstrand $(\bowtie) \cdot \AA$. Olson · M. Brandström Durling ·

M. Karlsson · J. Stenlid

Department of Forest Mycology and Plant Pathology,

Uppsala BioCenter, Swedish University of Agricultural Sciences,

Box 7026, 75007 Uppsala, Sweden

e-mail: kajsa.himmelstrand@slu.se forces for large mitochondrial genomes: the homing endonuclease genes in introns and integration of plasmid DNA.

Keywords Mitochondrial genome $\cdot$ Mitochondrial evolution $\cdot$ Pathogen $\cdot$ Homing endonuclease genes . Plasmid DNA

\section{Introduction}

Mitochondria are believed to have a monophyletic origin from an endosymbiotic $\alpha$-proteobacterium that was engulfed, more than one billion years ago, by a eukaryotic common ancestor (Gray et al. 2001; Bullerwell and Lang 2005; Koumandou et al. 2013). The mitochondrial (mt) genomes contain considerably fewer genes than freeliving $\alpha$-proteobacteria and many of the genes that are required for its function seem to have been transferred to the nucleus or replaced by already existing nuclear genes with similar function (Adams and Palmer 2003). Mitochondrial genomes occur either as circular or linear molecules (Burger et al. 2003).

Genes encoded by the mt genome can be divided into two groups: core genes and exchangeable genes. Core genes are involved in respiration, oxidative phosphorylation and translation (Burger et al. 2003), while exchangeable genes are characterised by variation in type and number among species. There are relatively few differences of $\mathrm{mt}$ core genes between and within kingdoms and phyla, although the exact compositions can vary (Adams and Palmer 2003). The exchangeable genes can be intronic genes, plasmid-derived genes and non-conserved open reading frames (ORFs). Mitochondrial plasmids are commonly found in plant and fungal mitochondria and are selfreplicating genetic elements that have little or no homology 
to the mt DNA, hence thought to have a separate evolutionary history from their hosts (Cahan and Kennell 2005; Formighieri et al. 2008). In Basidiomycetes, mt plasmids have been found in for example Flammulina velutipes (Nakai et al. 2000) and Pleurotus ostreatus (Yui et al. 1988) and integrated in $\mathrm{mt}$ genomes in Agaricus bisporus (Ferandon et al. 2013), P. ostreatus (Wang et al. 2008) and Moniliophthora perniciosa (Formighieri et al. 2008). The core genes in fungal $\mathrm{mt}$ genomes often contain introns with conserved RNA secondary structures involved in autocatalytic splicing (Lang et al. 2007). Many of these introns contain ORFs encoding homing endonuclease genes (HEGs) that can insert the intron into another intronless gene through a mechanism where the target gene is cleaved at rare recognition sites (Lang et al. 2007). This mechanism is called intron homing and could be the reason for the great variability of intron number between species. Some of the HEGs are also functioning as maturases that promote the RNA folding of the introns (Belfort 2003).

Mitochondrial sizes vary extensively in animals and plants with fungal $\mathrm{mt}$ sizes being intermediate $(12-236 \mathrm{~kb})$. At present, there are annotated $\mathrm{mt}$ genomes from 165 fungal species (http://www.ncbi.nlm.nih.gov/genomes/GenomesGr oup. .gi ?opt $=$ organelle $\&$ taxid $=4751 \&$ sort $=$ Genome). The fungal species investigated so far have a core set of 13-15 protein coding genes and two rRNA genes $(r n s, r n l)$ in common, although atp9 are lost in euascomycetes (Adams and Palmer 2003). Two other genes, $r p s 3$ and $r n p B$, are repeatedly lost in various fungal lineages (Adams and Palmer 2003; Bullerwell and Lang 2005). Seven genes that encode subunits of the NADH dehydrogenase complex are lost in the mt genomes of the genus Schizosaccharomyces and some Saccharomyces species (Bullerwell and Lang 2005).

In addition to energy production, the mitochondria play a fundamental role in metabolism, ion homeostasis, and apoptosis (Burger et al. 2003). The mitochondrion can also have a role in altering the virulence of fungal pathogens (Rogers et al. 1987; Monteiro-Vitorello et al. 1995; Ghabrial and Suzuki 2009; Ma et al. 2009; MonteiroVitorello et al. 2009). We have earlier shown that the mitochondrial origin influence virulence in Heterobasidion hybrids (Olson and Stenlid 2001).

Heterobasidion irregulare belong to the $H$. annosum sensu lato (s.l.) species complex in the phylum Basidiomycota, in the class of Agaricomycetes. Members of $\mathrm{H}$. annosum s.l. are severe necrotrophic root pathogens on conifers, with different but partially overlapping host ranges (Dalman et al. 2010). H. irregulare is distributed throughout the temperate regions of North America and has several Pinus and Juniperus tree species as its main hosts (Otrosina and Garbelotto 2010). The nuclear and mitochondrial genomes of $H$. irregulare strain TC 32-1 were recently sequenced (Olson et al. 2012).
The first objective of this study was to characterise the $\mathrm{mt}$ genome of $H$. irregulare and to describe its unique features. The second objective was to perform a comparative Basidiomycete $\mathrm{mt}$ genome study, to investigate the processes involved in the large variations of $\mathrm{mt}$ genome sizes in Basidiomycetes.

\section{Materials and methods}

Genome annotation and analysis

The H. irregulare strain TC 32-1 genome v.2 (http:// genome.jgi-psf.org/Hetan2/Hetan2.home.html) was used in the current work (Olson et al. 2012). The whole mt genome was obtained in one of the assembled contigs. Circularity was tested by performing several different assemblies that resulted in different overlapping start and end points of linear contigs. ORFs longer than $150 \mathrm{bp}$ were identified using ORFfinder, codon usage Table 4 (http://www.ncbi.nlm.nih. gov/projects/gorf/). ORFs were used to search the nonredundant NCBI database with BLASTP to annotate and find conserved genes.

Putative plasmid genes were defined as genes with similarity ( $E$ value $\leq 0.002$ ) to mitochondrial plasmid genes (integrated in $\mathrm{mt}$ genomes or not). Exon/intron boundaries of core genes were located by means of CLUSTALW alignment with homologous genes from other closely related fungal species. Predicted gene products from ORFs, $420 \mathrm{bp}$ or longer, with limited similarity ( $E$ value $>0.1$ ) to characterised proteins were categorised as non-conserved ORFs (ncORFs). The ORFs were required to start with a methionine codon. ORFs with lengths $150-420 \mathrm{bp}$, not overlapping with annotated genes, were categorised as small ORFs. Homing endonuclease genes (HEGs) in introns were predicted based on similarity with other HEGs, and were not required to start with a methionine codon and stop codons were admitted inside the genes.

InterProscan (Hunter et al. 2009) was used to search for protein signatures in ncORFs. The small and large subunit ( $r n s, r n l$ ) ribosomal RNA (rRNA) genes were identified with BLAST using genes from closely related species. The program tRNAscan-SE (Lowe and Eddy 1997) was used to identify the tRNA genes. The $H$. irregulare mt genome with annotations have been submitted to GenBank, accession number KF957635.

The frequency of codon usage was calculated for core genes, exchangeable genes and small ORFs and differences were studied with correspondence analysis (COA) using CodonW (Peden 1999). Small ORFs with similar codon usage as conserved genes were categorised as small hypothetical ORFs and were subjected to BLAST analysis against the NCBI nucleotide database. Eight genes from 
the nuclear genome of $H$. irregulare [F-type ATPase alpha (protein ID 35948), F-type ATPase b (147326), cytochrome c oxidase assembly protein 11 (383265), cytochrome c oxidase assembly protein 15 (34965), translation elongation factor 1a (406970), glyceraldehyde 3-phosphate dehydrogenase (419475), heat shock protein 90 (311260) and calmodulin (148960)] were retrieved as reference genes and included in the COA analysis. Differences in codon usage between genes were visualised by plotting the position of each gene on the resulting COA-axis 1 and 2.

Anticodons of the 26 tRNAs and the concatenated codon usage of the annotated genes were compared. Artemis (Rutherford et al. 2000) and DNA plotter (Carver et al. 2009) were used to visualise and calculate the $\mathrm{mt}$ genome, GC content, GC skew and cumulative GC skew.

\section{Basidiomycete mitochondrial genome comparison}

Nineteen thoroughly annotated Basidiomycete $\mathrm{mt}$ genomes present at the time of the study were retrieved from NCBI and EMBL (Table 1) (Paquin et al. 1997; Wang et al. 2008; Formighieri et al. 2008; Haridas and Gantt 2010; Stone et al. 2010; Yoon et al. 2012; Costa et al. 2012; Zhao et al. 2013; Ferandon et al. 2013; Hegedusova et al. 2014). The mt genome content of each species was divided into the following structural categories: (1) Electron transport and ATP synthesis genes, (2) rRNA genes, (3) tRNA genes, (4) Intergenic regions (without repetitive regions), (5) Repetitive regions,
(6) Introns (without intronic ORFs), (7) Intronic ORFs, (8) ncORFs, (9) Putative plasmid genes and (10) The ribosomal protein gene rps3. Sequence lengths were calculated for each category using Artemis. In order to get comparable data between the species, all ncORFs were defined as ORFs with a length over a limit where the probability of finding such an ORF by chance was equal to finding a 420 bp ORF in $H$. irregulare, given the base composition of the species. Repeats were detected in the species with the program Repseek (Achaz et al. 2007) using a cutoff $p$ value of 0.01 . Some additional annotation of the intronic ORFs was done in Cryptococcus neoformans, Phakopsora meibomiae, P. pachyrhizi, Trametes cingulata, Tilletia indica, T. walkeri, Ustilago maydis, Phlebia radiata, Lentinula edodes, M. roreri, Cantharellus cibarius, F. velutipes, A. bisporus, and Ganoderma luci$d u m$. The median differences of $m t$ genome lengths between the Agaricomycete group and the other Basidiomycetes were tested with the Wilcoxon rank-sum test with the null hypothesis no difference in length.

\section{Results}

Mitochondrial genome structure and annotation of $H$. irregulare

The circular 114, $193 \mathrm{bp}$ long $\mathrm{mt}$ genome of $H$. irregulare had a mean GC content of $22.8 \%$. Fifteen protein coding

Table 1 The 20 annotated Basidiomycete mitochondrial genomes used in the current study

\begin{tabular}{|c|c|c|c|}
\hline Species & Class & Order & Accession number \\
\hline Pleurotus ostreatus & Agaricomycetes & Agaricales & NC_009905 \\
\hline Schizophyllum commune & Agaricomycetes & Agaricales & NC_003049 \\
\hline Moniliophthora perniciosa & Agaricomycetes & Agaricales & NC_005927 \\
\hline Moniliophthora roreri & Agaricomycetes & Agaricales & NC_015400 \\
\hline Lentinula edodes & Agaricomycetes & Agaricales & NC_018365.1 \\
\hline Flammulina velutipes & Agaricomycetes & Agaricales & NC_021373 \\
\hline Agaricus bisporus & Agaricomycetes & Agaricales & JX271275 \\
\hline Heterobasidion irregulare & Agaricomycetes & Russulales & KF957635 \\
\hline Trametes cingulata & Agaricomycetes & Polyporales & NC_013933 \\
\hline Phlebia radiata & Agaricomycetes & Polyporales & NC_020148 \\
\hline Ganoderma lucidum & Agaricomycetes & Polyporales & HF570115 \\
\hline Cantharellus cibarius & Agaricomycetes & Cantharellales & NC_020368 \\
\hline Cryptococcus neoformans var. grubii & Tremellomycetes & Tremellales & NC_004336 \\
\hline Ustilago maydis & Ustilaginomycetes & Ustilaginales & NC_008368 \\
\hline Tilletia indica & Exobasidiomycetes & Tilletiales & NC_009880 \\
\hline Tilletia walkeri & Exobasidiomycetes & Tilletiales & NC_010651 \\
\hline Jaminaea angkoriensis & Exobasidiomycetes & Microstromatales & NC_023248 \\
\hline Phakopsora meibomiae & Urediniomycetes/pucciniomycetes & Uredinales & NC_014352 \\
\hline Phakopsora pachyrhizi & Urediniomycetes/pucciniomycetes & Uredinales & NC_014344 \\
\hline Rhodotorula taiwanensis & Urediniomycetes & Sporidiales & HF558455 \\
\hline
\end{tabular}


core genes, 28 RNA genes, six ncORFs, 36 small hypothetical ORFs, 14 intronic ORFs, two putative plasmid genes, two putative dpo pseudogenes and one partial gene were identified (Fig. 1).

Seven out of the 15 core genes were predicted to encode proteins belonging to the NADH dehydrogenase complex (nad1, nad2, nad3, nad4, nad4L, nad5, nad6), one gene product belonged to the cytochrome bc1 complex ( $c o b)$, three to the cytochrome c oxidase complex ( $\operatorname{cox} 1$, cox2, cox3) and three to the ATP synthase complex (atp6, atp8, atp9). One additional partial $n a d 2$ gene was also identified.
The last core gene was predicted to encode the ribosomal small subunit protein 3 (rps3), which is involved in ribosome assembly. The identified RNA genes included the small and large subunit rRNA genes ( $r n s$ and $r n l)$ and 26 tRNAs. Two ORFs with similarity to each other $(E$ value $6 \mathrm{e}^{-17}$ ) were annotated as putative plasmid genes ( $p p l l$ and ppl2), since they show similarity with putative plasmid proteins from $P$. ostreatus and $M$. perniciosa ( $E$ values $3 \mathrm{e}^{-12}$ and $3 \mathrm{e}^{-7}$ ). Two putative plasmid pseudo-B-type DNA polymerase genes ( $p s-d p o 1$ and $p s-d p o 2$ ) containing frame shifts were found next to one of the putative plasmid genes.

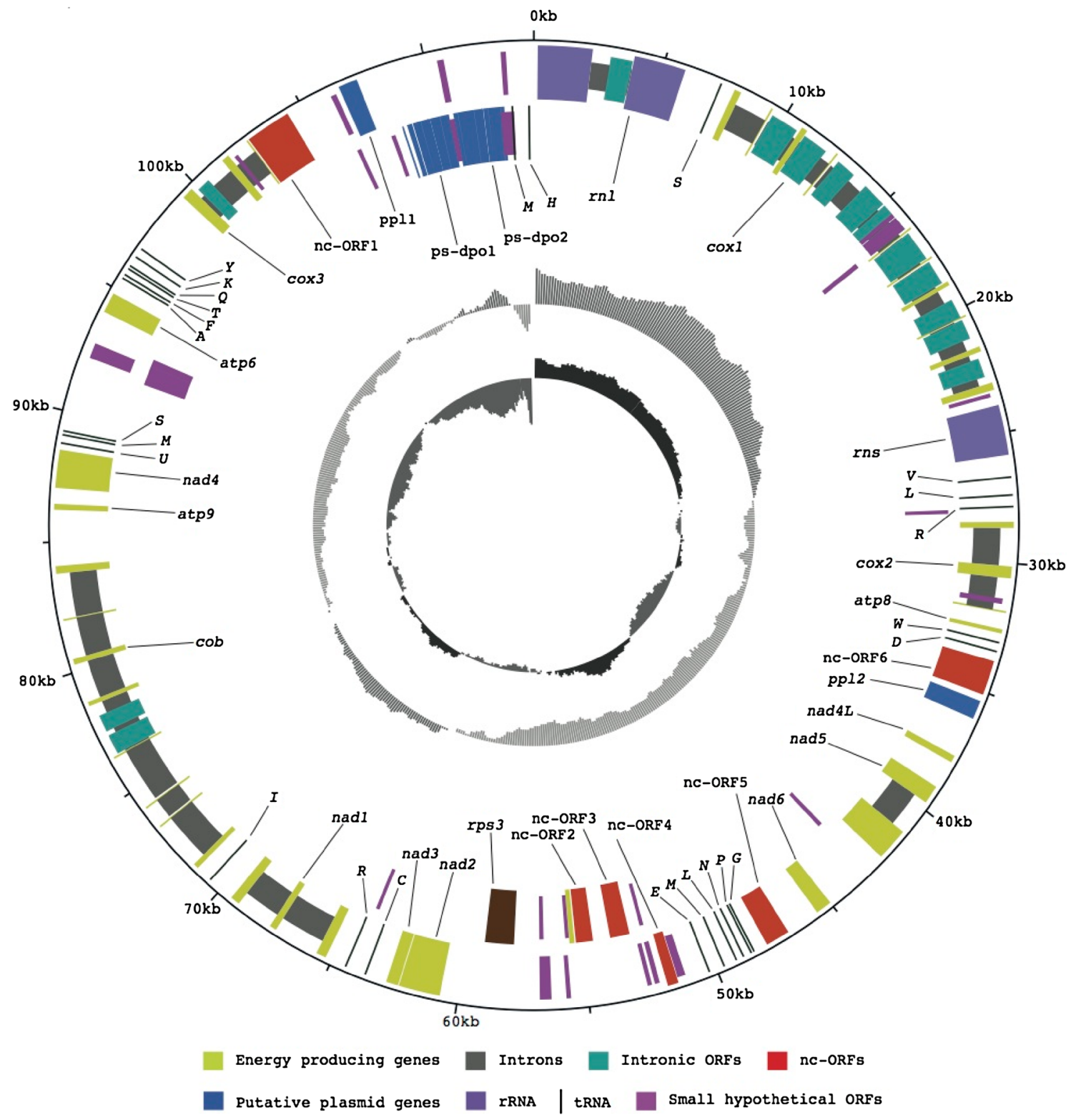

Fig. 1 Map of the Heterobasidion irregulare mitochondrial genome. The boxes in light green are the energy producing genes, rps 3 is brown, the turquoise boxes are the intronic genes, black lines represent tRNAs, the non-conserved ORFs are red-brown and the blue are the putative plasmid and pseudo-dpo genes. The different rings, from the outside and in: (i) genes in the clockwise direction. (ii) Genes in the anticlockwise direction. (iii) GC content. (iv) GC skew 
In five of the six large ncORFs (ncORF1-6), transmembrane regions were found. Four of the ncORFs were located adjacent to each other, while two additional ncORFs were neighbouring putative plasmid genes (Fig. 1). The ncORFs had no similarity to each other.

In two regions, genes were predicted to be transcribed on the complementary strand compared to the majority of genes: one region with two of the four ncORFs adjacent to each other, the partial nad2 gene and rps3. The other complementary strand region contains the two putative pseudodpo genes. The cumulative GC skew has a minimum value upstream of the $r n l$ gene and is therefore considered as the putative location of the origin of replication for the $H$. irregulare mt genome (Touchon et al. 2005; Formighieri et al. 2008).

Introns and intronic ORFs in H. irregulare

The 24 introns found were unevenly distributed among the core genes: nine in coxl, two in cox2, two in cox3, seven in cob, two in nadl, one in nad5 and one in $r n l$ (Fig. 1). In ten of these introns, 14 intronic ORFs were found. Ten were found in the introns of coxl, one in cox3, two in $c o b$ and one in $r n l$. There were as many as three intronic ORFs in intron four of coxl, whereof one was a putative pseudointronic ORF since it contained frame shifts. BLAST searches showed that the intronic ORFs were predicted to encode conserved homing endonuclease genes (HEGs, $E$ values between $2 \mathrm{e}^{-30}$ and $1 \mathrm{e}^{-132}$ ). All but one intronic ORF contained one of the two HEG motifs: LAGLIDADG or GIY-YIG. The best BLAST hit of the HEGs was always a HEG in a homologous core gene intron but not necessary in a phylogenetically closely related fungus. Eight of the intronic ORFs were found in the same reading frame as the upstream exon, ten did not start with the typical methionine codon and three had internal stop codons in conserved sequences.

The tRNAs and codon usage in $H$. irregulare

In total, 26 genes for tRNA was identified, clustered in nine regions. All but two tRNAs were clustered with two to six tRNAs in each group. Two tRNAs located between a pseudo-dpo gene and $r n l$ were transcribed on the opposite strand (Fig. 1). For every amino acid there was one or more corresponding tRNA. However, the most used codons did not always have a corresponding tRNA in the mt genome even when the wobbling rule was taken into account (Crick 1966), (http://www.ncbi.nlm.nih.gov/books/NBK21424/). The following codons were among the most frequently used but did not have the corresponding tRNA in the $\mathrm{mt}$ genome: GCT (Ala), GGT (Gly), ATA (Ile/Met), CCT (Pro), TCT (Ser), ACT (Thr) and GTT (Val).
Codon usage was analysed for all core and exchangeable genes including all small ORFs. There was a strong codon bias towards using AT-rich codons in all genes and the small ORFs. The tRNAs, on the other hand, did not show the same bias towards AT-rich anticodons. Instead, the $5^{\prime}$ base of the anti codons strongly favoured primarily a $\mathrm{T}$ and secondarily a G: 22 of the 25 anticodons started with either a $\mathrm{T}$ or a $\mathrm{G}$.

Codon usage analysis with the annotated genes in the $\mathrm{mt}$ genome and eight nuclear genes clearly separated $\mathrm{mt}$ genome genes from nuclear genes along the first axis in a COA (data not shown). In the COA analysis of codon usage in annotated genes and the small ORFs, two groups were formed (Fig. 2a). One group consisted of all of annotated genes except one intronic ORF, the ncORFs along with some of the small ORFs. The remaining small ORFs were located in the second group on the negative side of axis 1 .

When performing the COA excluding small ORFs, two distinct groups were formed (Fig. 2b). One group consisted of genes predicted to encode proteins involved in energy production, while the other group consisted of genes that were not predicted to be involved in energy production. There were 11 codons that differed significantly in usage between the two groups: Leu UUA, Val GUA, Tyr UAU, Glu GAA $(p<0.01)$ and Phe UUC, Ile AUA, Gln CAA, Asp GAU, Ser UCA, Pro CCA, Arg AGA $(0.01<p<0.05)$. Eight of these 11 codons had a corresponding tRNA in the mt genome (Fig. 3).

Comparative analysis of Basidiomycete mitochondrial genomes

To analyse the relative contribution in size of different $\mathrm{mt}$ genome parts for Basidiomycetes, $19 \mathrm{mt}$ genomes (Table 2) were re-annotated in a consistent way. This resulted in the identification of rps3, previously not reported, in the two Tilletia species. In addition, some of the exchangeable genes were in unusual positions. There were HEGs in intergenic areas in T. indica, M. perniciosa, and G lucidum and putative plasmid genes in introns in T. cingulata and $A$. bisporus. All the $\mathrm{mt}$ genomes including $H$. irregulare had the same set of core genes: nad1, nad2, nad3, nad4, nad4L, nad5, nad6, cob, cox1, cox2, cox3, atp6, atp8, atp9, rps3, $r n s$ and $r n l$. However, the $P$. radiata $\mathrm{mt}$ genome contained two nad6 genes due to a 6,077 bp long duplication. Putative plasmid genes were found in all the 12 Agaricomycete $\mathrm{mt}$ genomes and ncORFs in 11 of them. There was a large variation in the number and positions of putative plasmid genes and ncORFs in the various mt genomes. In M. perniciosa, the six putative plasmid genes were clustered in the same location and five of them were within two inverted repeats typical for $\mathrm{mt}$ plasmids. There were three ncORFs in the same area (Formighieri et al. 2008). In the closely 
Fig. 2 Correspondence analysis of codon usage of mitochondrial genes in $H$. irregulare. Correspondence analysis of codon usage was performed on annotated genes, putative genes including small ORFs (a) and without the small ORFs (b) identified in the $\mathrm{mt}$ genome of $H$. irregulare, using the program CodonW

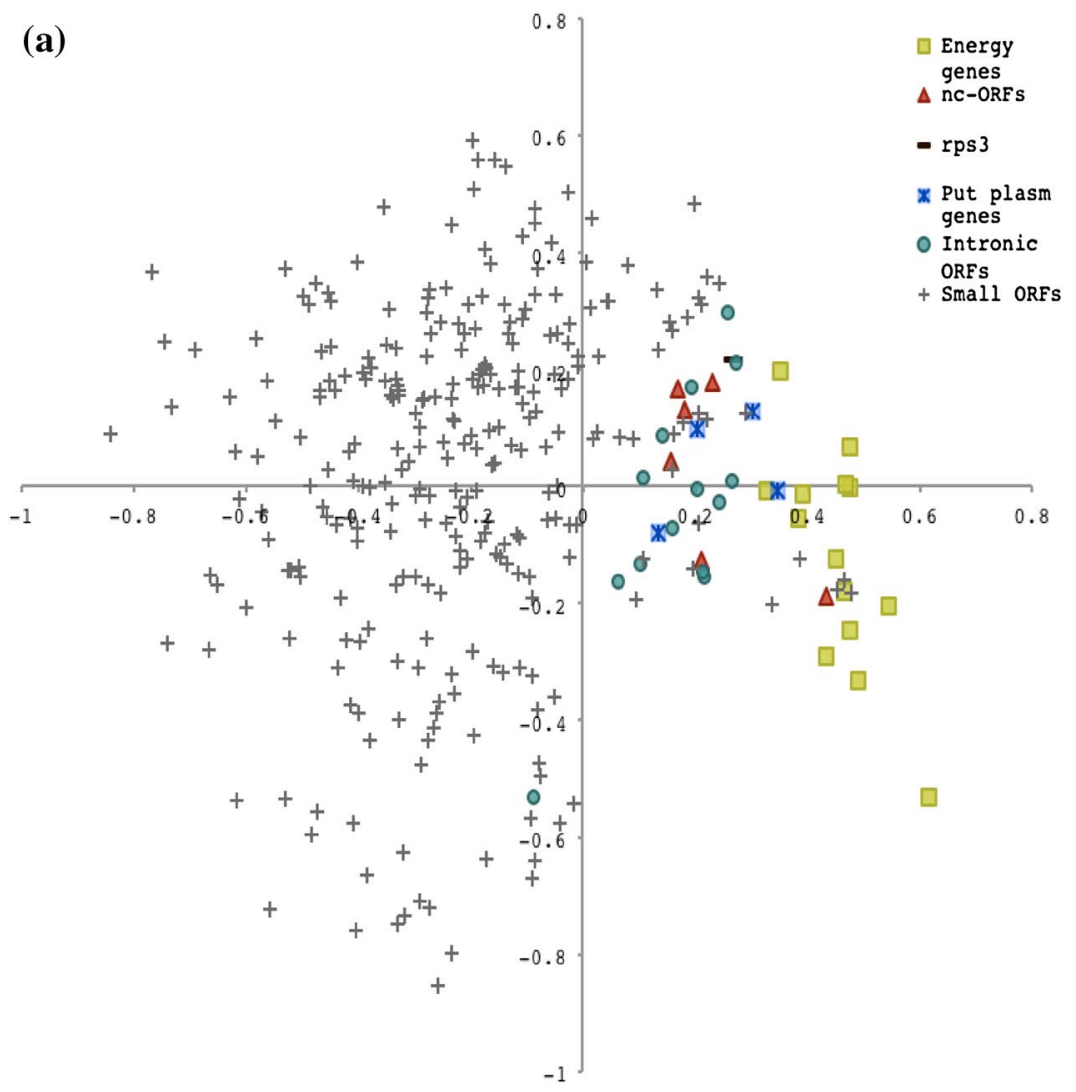

(b)

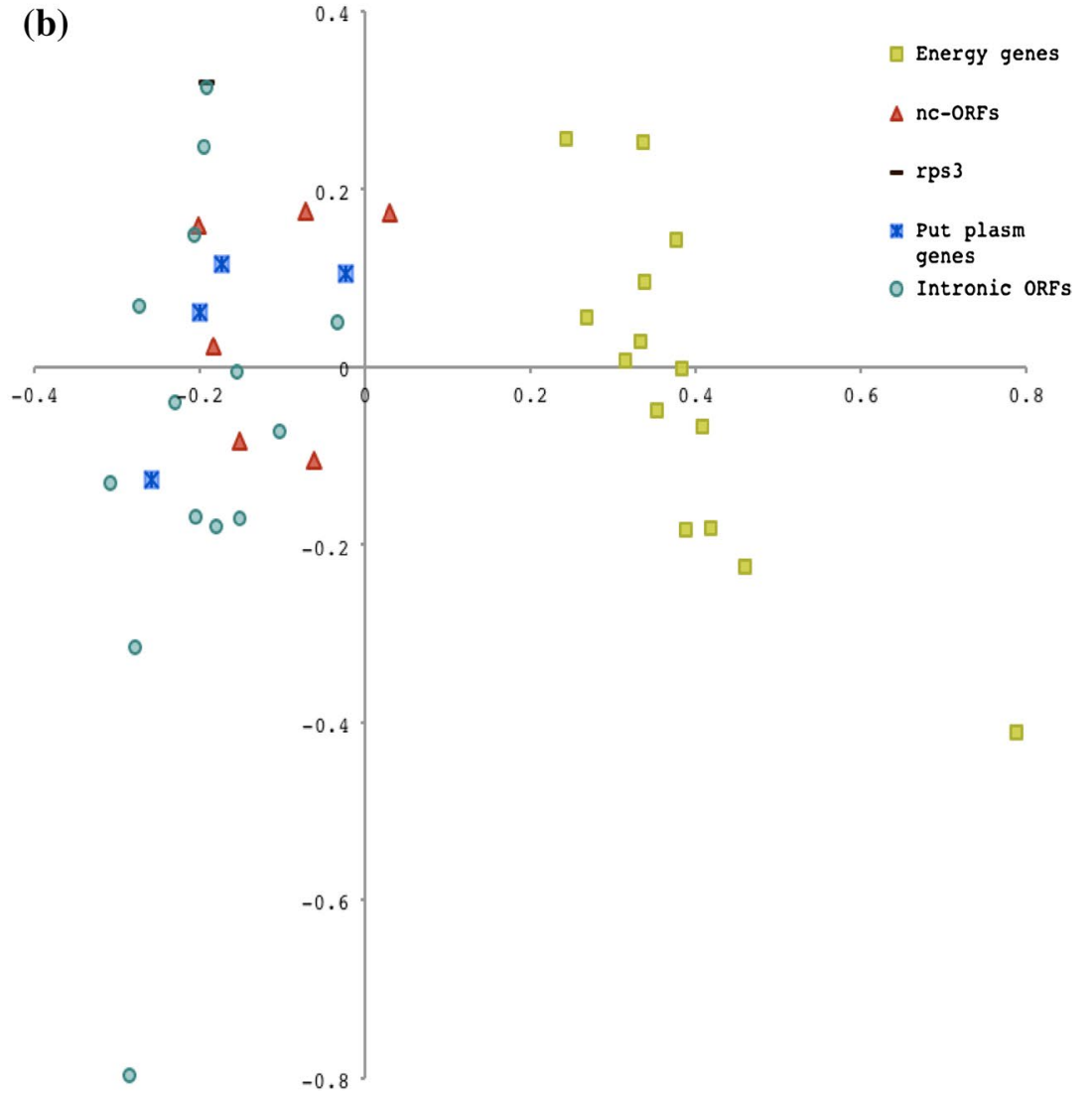




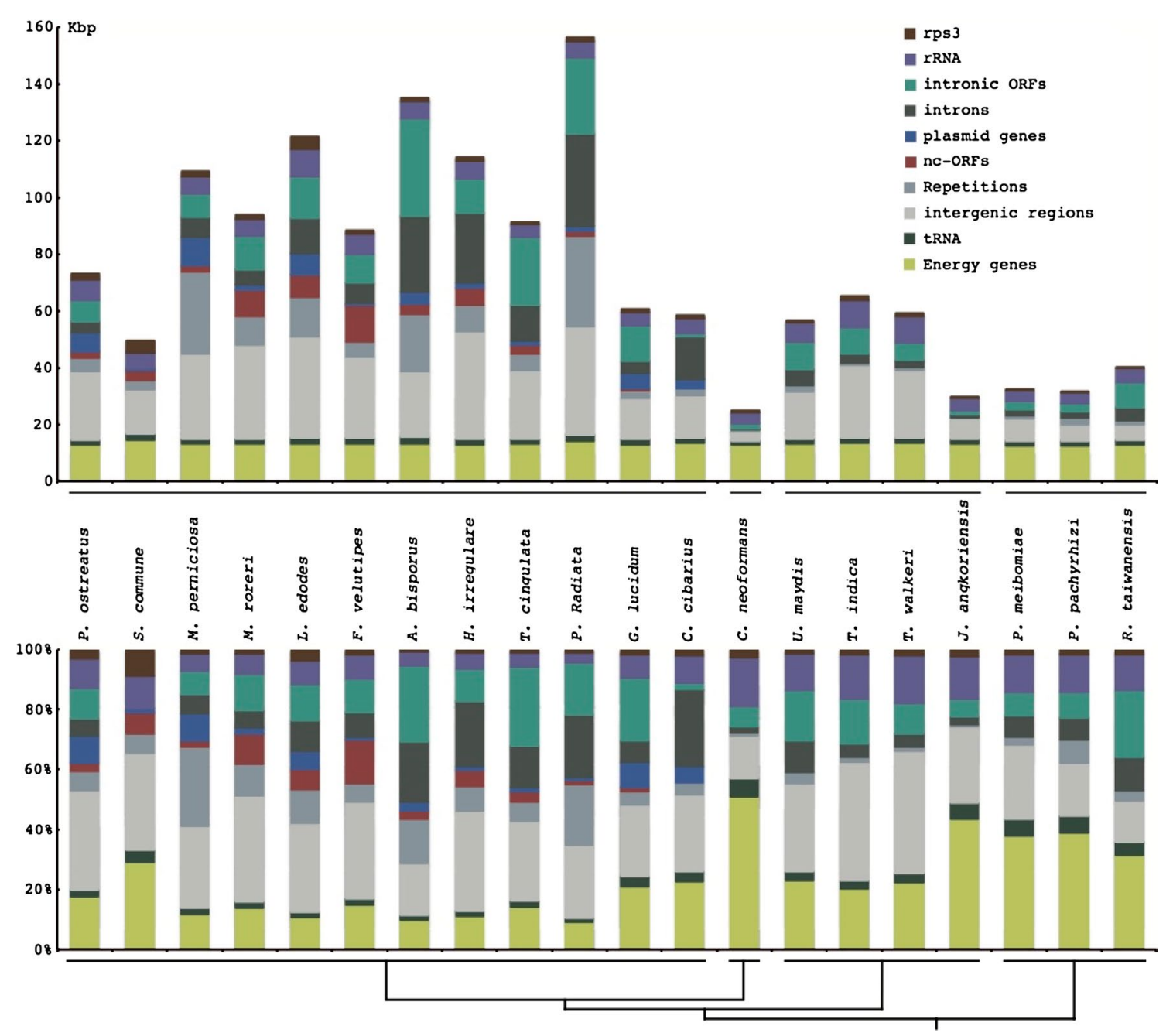

Fig. 3 Comparison of Basidiomycete mitochondrial genome size differences, separated into different categories indicated in the diagram. The total DNA amount corresponding to each category in each genome (top panel) and the percentages of the categories for each $\mathrm{mt}$ genome (bottom panel) is plotted. A schematic representation of the taxonomic relationships of the included species is given related species $M$. roreri, a $14 \mathrm{~Kb}$ area between nad 3 and nadl was found with five ncORFs and two putative plasmid genes (according to our criteria) (Costa et al. 2012). Agaricus bisporus had two large inverted repeats and each of them contained a remnant copy of a DNA polymerase gene (Ferandon et al. 2013). Between the inverted repeats there were also two putative plasmid genes and two ncORFs plus the genes $r p s 3, r n l, n a d 3$ and $n a d 2$. There were three areas in A. bisporus where putative plasmid genes and ncORFs were clustered together. The putative plasmid gene in $F$. velutipes was clustered together with three ncORFs. In the $H$. irregulare $\mathrm{mt}$ genome, three of the four putative plasmid genes were clustered together with an ncORF. The four putative plasmid genes in $C$. cibarius were all clustered in the same area. In the other six Agaricomycete mt genomes, the putative plasmid genes and ncORFs were more evenly distributed. The amount of repetitive areas differed between the species, whereof $M$. perniciosa and $P$. radiata were the most outstanding with a repetitive content of 26.5 and $20.3 \%$, respectively. The repetitive regions were also the most variable areas with a coefficient of variation of 1.27 followed by introns (without intronic ORFs), intronic ORFs, putative plasmid genes, ncORFs, rps 3 and intergenic regions (Table 3 ). The energy production genes had by far the smallest coefficient of variation (0.04). The nine largest $\mathrm{mt}$ genomes among the studied Basidiomycetes all belonged to the Agaricomycetes and all nine contained both introns with intronic ORFs and putative plasmid genes together with ncORFs. $P$. radiata had the largest $\mathrm{mt}$ genome (156,348 bp) and the largest areas of intergenic regions $(69,951 \mathrm{bp})$, introns without intronic ORFs (32,536 bp) and repetitive sequences (31,805 bp) mostly found in the intergenic regions. The A. bisporus $\mathrm{mt}$ genome $(135,005 \mathrm{bp})$ had the highest number of introns (46) and 
Table 2 Characteristics of the Basidiomycete mitochondrial genomes used in the current study

\begin{tabular}{|c|c|c|c|c|c|c|c|c|c|}
\hline Species & Length (bp) & $\begin{array}{l}\text { GC content } \\
(\%)\end{array}$ & $\begin{array}{l}\text { ncORF cut off } \\
\text { (bp) }\end{array}$ & $\begin{array}{l}\text { Repetitive areas } \\
(\%)\end{array}$ & tRNA & Intronic ORFs & Introns & ncORFs & Plasmid genes \\
\hline P. ostreatus & 73,242 & 26.4 & 460 & 6.51 & 24 & 9 & 12 & 2 & 3 \\
\hline S. commune & 49,704 & 21.9 & 410 & 6.48 & 27 & 0 & 0 & 4 & 1 \\
\hline M. perniciosa & 109,103 & 31.9 & 540 & 26.54 & 26 & 9 & 13 & 8 & 6 \\
\hline M. roreri & 93,722 & 27.6 & 480 & 10.72 & 26 & 12 & 12 & 9 & 2 \\
\hline L. edodes & 121,394 & 30.7 & 525 & 11.37 & 28 & 15 & 19 & 7 & 3 \\
\hline F. velutipes & 88,508 & 16.5 & 355 & 6.00 & 26 & 10 & 10 & 7 & 1 \\
\hline A. bisporus & 135,005 & 29.1 & 500 & 14.78 & 35 & 37 & 46 & 4 & 6 \\
\hline H. irregulare & 114,193 & 22.8 & 420 & 8.17 & 26 & 14 & 24 & 6 & 2 \\
\hline T. cingulata & 91,500 & 24.5 & 440 & 6.24 & 25 & 28 & 26 & 4 & 1 \\
\hline P. radiata & 156,348 & 31.2 & 530 & 20.34 & 28 & 28 & 37 & 2 & 1 \\
\hline G. lucidum & 60,630 & 26.7 & 465 & 4.39 & 28 & 14 & 13 & 1 & 3 \\
\hline C. cibarius & 58,656 & 26.8 & 470 & 4.20 & 26 & 13 & 13 & 0 & 4 \\
\hline C. neoformans & 24,874 & 35.0 & 600 & 1.00 & 21 & 2 & 2 & 0 & 0 \\
\hline U. maydis & 56,814 & 31.2 & 530 & 3.71 & 23 & 12 & 14 & 0 & 0 \\
\hline T. indica & 65,147 & 28.9 & 500 & 1.54 & 24 & 11 & 11 & 0 & 0 \\
\hline T. walkeri & 59,352 & 28.8 & 500 & 1.30 & 24 & 7 & 9 & 0 & 0 \\
\hline J. angkoriensis & 29,999 & 32.2 & 545 & 0.75 & 22 & 2 & 2 & 0 & 0 \\
\hline P. meibomiae & 32,520 & 34.9 & 595 & 2.76 & 24 & 3 & 5 & 0 & 0 \\
\hline P. pachyrhizi & 31,825 & 34.6 & 590 & 7.75 & 24 & 3 & 5 & 0 & 0 \\
\hline R. taiwanensis & 40,392 & 41.0 & 730 & 3.30 & 23 & 8 & 13 & 0 & 0 \\
\hline
\end{tabular}

Table 3 Variation in length of the different categories and the repetitive regions, in the form of standard deviation and coefficient of variation

\begin{tabular}{lrrl}
\hline Category & \multicolumn{1}{l}{ Mean } & $\begin{array}{l}\text { Standard } \\
\text { deviation }\end{array}$ & $\begin{array}{l}\text { Coefficient of } \\
\text { variation }\end{array}$ \\
\hline Repeat regions & $7,354.80$ & $9,365.86$ & 1.27 \\
Introns $^{\mathrm{a}}$ & $9,272.95$ & $9,372.85$ & 1.01 \\
Intronic ORFs $^{\mathrm{a}}$ & $10,852.26$ & $8,944.20$ & 0.82 \\
Plasmid genes $^{\mathrm{a}}$ & $3,685.67$ & $3,008.70$ & 0.82 \\
ncORFs $^{\mathrm{a}}$ & $4,833.82$ & $3,790.91$ & 0.78 \\
rps3 $_{\text {Intergenic regions }}$ & $1,426.80$ & $1,084.65$ & 0.76 \\
rRNA & $28,200.15$ & $18,721.15$ & 0.66 \\
tRNA & $6,021.95$ & $1,802.27$ & 0.30 \\
Energy genes & $1,911.30$ & 230.84 & 0.12 \\
\hline
\end{tabular}

a Species missing this category were excluded from the analysis

the largest areas of intronic ORFs (34,057 bp), whereas $L$. edodes (121,394 bp) had larger areas of intergenic regions (49,586 bp), ncORFs (7,264 bp) and putative plasmid genes (7,264 bp) and H. irregulare (114,193 bp) had larger areas with introns $(24,921 \mathrm{bp})$. M. perniciosa $(109,103 \mathrm{bp})$ had the largest areas with putative plasmid genes $(7,264 \mathrm{bp})$ and also large areas with intergenic $(58,832 \mathrm{bp})$ and repetitive regions $(28,951 \mathrm{bp})$ in comparison with the other $\mathrm{mt}$ genomes. $F$. velutipes $(88,508 \mathrm{bp})$ had the largest areas of ncORFs $(12,807 \mathrm{bp})$. The Ustilaginomycete and Exobasidiomycete species had medium $\mathrm{mt}$ genome sizes (57-65 $\mathrm{kbp}$ ), with the exception for $J$. angkoriensis, the three Urediniomycetes species and $C$. neoformans all had small $\mathrm{mt}$ genome sizes $(<40 \mathrm{kbp})$. The Agaricomycete genomes were significantly larger than other Basidiomycetes $(p=0.00024)$.

\section{Discussion}

The $114 \mathrm{~kb}$ long $H$. irregulare $\mathrm{mt}$ genome is one of the largest sequenced and annotated fungal $\mathrm{mt}$ genomes. Heterobasidion irregulare belongs to the class Agaricomycete that contains a majority of the species with the largest fungal mt genomes known to date. All of the largest Agaricomycete mt genomes have long regions with intergenic areas, ncORFs and putative plasmid genes whereas some also have long regions with introns and intronic ORFs. Variable $\mathrm{mt}$ genome sizes have previously been attributed to intergenic regions, introns and in some cases extensive tandem repeat arrays or stem loop motifs (Burger et al. 2003). Our observations show that variation in exchangeable gene content and repeat regions are also important contributors to differences in $\mathrm{mt}$ genome size. 
Many fungal and plant species have mt plasmid DNA integrated in their $\mathrm{mt}$ genomes, despite the fact that no genes coding for integrase activity has been found in $\mathrm{mt}$ plasmids so far (Cahan and Kennell 2005). All the mt genomes of Agaricomycetes contain putative plasmid genes and ncORFs, with the exception for $C$. cibarius where the mt genome lacks ncORFs. However, both putative plasmid genes and ncORFs are absent in the other studied Basidiomycete classes. Our data suggests that the presence of ncORFs in $\mathrm{mt}$ genomes is somehow linked to mt plasmids based on the following observations: (1) The simultaneous occurrence of putative plasmid genes and ncORFs in mt genomes. (2) NcORFs and putative plasmid genes are often clustered in several of the mt genomes. (3) The ncORFs have the same preferential codon usage as the putative plasmid genes in $H$. irregulare. Given the coexistence of ncORFs and putative plasmid genes in Agaricomycetes, the lack of ncORFs in the $C$. cibarius $\mathrm{mt}$ genome most likely represents a loss of ncORFs in the ancestor of C. cibarius. The apparent connection may suggest that the ncORFs originate from an mt plasmid, or alternatively that mt plasmids may enable integration of non-mitochondrial DNA into the mt genome. The different patterns of codon usage in the putative plasmids and ncORFs, compared to the core genes, may indicate separate evolutionary origins of these two classes of genes. Since putative plasmid genes occurred exclusively in the Agaricomycetes, we hypothesise that the common ancestor of the Agaricomycetes acquired the mt plasmids. The acquisition might have been initiated by the uptake of a plasmid into the mitochondria in the Agaricomycete ancestor, which was subsequently integrated into the $\mathrm{mt}$ genomes at several, independent occasions. A single integration event in the Agaricomycete ancestor is less likely since there is a considerable variation in the number and positioning of the putative plasmid genes among the Agaricomycete species.

HEGs have previously been proposed to go through a cyclical model of invasion, degeneration and loss where the HEGs only are selected for during the invasion phase. Nonetheless, some HEGs have been reported to persist over longer evolutionary times (Goddard and Burt 1999; Gogarten and Hilario 2006).

All Basidiomycete mt genomes except $S$. commune contained introns and intronic ORFs. However, the length of these regions varied largely among species with the largest regions in the Agaricomycete species. In this study, we found that a particular HEG of $H$. irregulare always showed the highest similarity to an HEG in an intron of a homologous core gene of another species, although it was not necessarily the closest related species that contained the most similar HEGs. This is in accordance with previous findings that group I introns have a preference to be inserted into highly conserved genes, such as $\operatorname{coxl}$ and $\operatorname{cob}$ (Paquin et al.
1997). Furthermore, between species, the intron movement nearly always occurs into the homologous gene site (Paquin et al. 1997; Haugen et al. 2005). Phylogenetic analyses suggest that horizontal gene transfer has occurred frequently but the mechanism of how the mobile introns are transferred between species barriers is not known (Goddard and Burt 1999; Wang et al. 2008). In H. irregulare, the intronic ORFs had a different preferential codon usage than the energy genes. The most likely explanation to this is that the intronic ORFs have a different evolutionary origin than the energy genes, possibly acquired through horizontal gene transfer. Alternatively, differences in expression levels can also result in differences in preferred codon usage. However, no study of intronic ORF gene expression is reported from $H$. irregulare.

Recently, an invasion of HEGs and self-splicing introns was shown to explain the large mt genome size of Rhynchosporium agropyri and $R$. secalis (Torriani et al. 2014). Similar expansions of mobile introns are reported from other ascomycete fungi including Fusarium spp. (Al-Reedy et al. 2012), Penicillium spp. and Aspergillus spp. (Joardar et al. 2012). The reoccurring internal stop codons and frame shifts and the varying number of HEGs in the studied species suggest that HEG and intron expansions can contribute to large $\mathrm{mt}$ genome sizes also in Basidiomycetes as previously reported for ascomycete fungi.

The presence of long ncORFs in fungal $\mathrm{mt}$ genomes with no similarity to genes in other species is common, and can contribute to a large $\mathrm{mt}$ genome size (Al-Reedy et al. 2012). However, it is difficult to confirm that these are authentic genes in the absence of homologous genes from other species. The ncORFs found in $T$. cingulata do not utilise the same codons as the core genes and were proposed not to correspond to authentic genes (Haridas and Gantt 2010). Since stop codons are AT rich, the probability for ncORFs larger than $420 \mathrm{bp}$ to occur by chance in a low GC content mt genome such as in H. irregulare is less than $7.5 \mathrm{e}^{-15}$. The fact that the ncORFs displayed the same preferential codon usage as conserved exchangeable genes, and the presence of several predicted transmembrane domains, suggests that the identified ncORFs are indeed authentic genes that could possibly originate from $\mathrm{mt}$ plasmids. On the other hand, 14 ncORFs with the same preferential codon usage as the core genes were found in M. perniciosa (Formighieri et al. 2008), which prompted the authors to suggest that the ncORFs represent functional genes that originate from the $\alpha$-proteobacterial ancestor. However, the ncORF in the G. lucidum mt genome found in this study was not expressed in a recent transcriptome analysis $(\mathrm{Li}$ et al. 2013).

We identified two independent factors as the driving forces for large mt genomes among Basidiomycetes. The first factor was the HEGs in the introns that are able to 
make the introns mobile and able to spread into intronless genes. The second factor was plasmid regions that are integrated in $\mathrm{mt}$ genomes, possibly leaving plasmid gene remnants in the form of ncORFs and larger intergenic areas. The $H$. irregulare isolate in this study was one of the parents in the interspecies crossing study that showed a connection between the mitochondria and virulence (Olson and Stenlid 2001). Possible factors that could have an influence on virulence are probably not the core genes of the $\mathrm{mt}$ genome but rather the exchangeable parts. We are now well positioned to continue this investigation by comparing the $\mathrm{mt}$ genome of $H$. irregulare with $H$. occidentale, the other parent in the hybrid study (Olson and Stenlid 2001).

Acknowledgments This project was financially supported by The Swedish Research Council for Environmental, Agricultural and Spatial Planning (FORMAS) and The Swedish Foundation for Strategic Research (SSF). We are also thankful to the US Department of Energy, Joint Genome Institute (JGI) for the sequencing and assembly work.

Open Access This article is distributed under the terms of the Creative Commons Attribution License which permits any use, distribution, and reproduction in any medium, provided the original author(s) and the source are credited.

\section{References}

Achaz G, Boyer F, Rocha EPC et al (2007) Repseek, a tool to retrieve approximate repeats from large DNA sequences. Bioinformatics 23:119-121

Adams KL, Palmer JD (2003) Evolution of mitochondrial gene content: gene loss and transfer to the nucleus. Mol Phylogenet Evol 29:380-395

Al-Reedy RM, Malireddy R, Dillman CB, Kennell JC (2012) Comparative analysis of Fusarium mitochondrial genomes reveals a highly variable region that encodes an exceptionally large open reading frame. Fungal Genet Biol 49:2-14

Belfort M (2003) Two for the price of one: a bifunctional intronencoded DNA endonuclease-RNA maturase. Genes Dev 17:2860-2863

Bullerwell CE, Lang BF (2005) Fungal evolution: the case of the vanishing mitochondrion. Curr Opin Microbiol 8:362-369

Burger G, Gray MW, Lang BF (2003) Mitochondrial genomes: anything goes. Trends Genet 19:709-716

Cahan P, Kennell JC (2005) Identification and distribution of sequences having similarity to mitochondrial plasmids in mitochondrial genomes of filamentous fungi. Mol Gen Genomics 273:462-473

Carver T, Thomson N, Bleasby A et al (2009) DNAPlotter: circular and linear interactive genome visualization. Bioinformatics 25:119-120

Costa GGL, Cabrera OG, Tiburcio RA et al (2012) The mitochondrial genome of Moniliophthora roreri, the frosty pod rot pathogen of cacao. Fungal Biol 116:551-562

Crick FHC (1966) Codon-anticodon pairing: the wobble hypothesis. J Mol Biol 19:548-555

Dalman K, Olson Å, Stenlid J (2010) Evolutionary history of the conifer root rot fungus Heterobasidion annosum sensu lato. Mol Ecol 19:4979-4993
Ferandon C, Xu J, Barroso G (2013) The $135 \mathrm{kbp}$ mitochondrial genome of Agaricus bisporus is the largest known eukaryotic reservoir of group I introns and plasmid-related sequences. Fungal Genet Biol 55:85-91

Formighieri EF, Tiburcio RA, Armas ED et al (2008) The mitochondrial genome of the phytopathogenic basidiomycete Moniliophthora perniciosa is $109 \mathrm{~kb}$ in size and contains a stable integrated plasmid. Mycol Res 112:1136-1152

Ghabrial SA, Suzuki N (2009) Viruses of plant pathogenic fungi. Annu Rev Phytopathol 47:353-384

Goddard MR, Burt A (1999) Recurrent invasion and extinction of a selfish gene. Proc Natl Acad Sci USA 96:13880-13885

Gogarten JP, Hilario E (2006) Inteins, introns, and homing endonucleases: recent revelations about the life cycle of parasitic genetic elements. BMC Evol Biol 6:94

Gray MW, Burger G, Lang BF (2001) The origin and early evolution of mitochondria. Genome Biol 2:REVIEWS1018

Haridas S, Gantt J (2010) The mitochondrial genome of the wooddegrading basidiomycete Trametes cingulata. FEMS Microb Lett 308:29-34

Haugen P, Simon DM, Bhattacharya D (2005) The natural history of group I introns. Trends Genet 21:111-119

Hegedusova E, Brejova B, Tomaska L et al (2014) Mitochondrial genome of the basidiomycetous yeast Jaminaea angkorensis. Curr Genet 60:49-59. doi:10.1007/s00294-013-0410-1

Hunter S, Apweiler R, Attwood TK et al (2009) InterPro: the integrative protein signature database. Nucl Acid Res 37:D211-D215

Joardar V, Abrams NF, Hostetler J et al (2012) Sequencing of mitochondrial genomes of nine Aspergillus and Penicillium species identifies mobile introns and accessory genes as main sources of genome size variability. BMC Genomics 13:698

Koumandou VL, Wickstead B, Ginger ML et al (2013) Molecular paleontology and complexity in the last eukaryotic common ancestor. Crit Rev Biochem Mol Biol 48:373-396

Lang B, Laforest M, Burger G (2007) Mitochondrial introns: a critical view. Trends Genet 23:119-125

Li J, Zhang J, Chen H et al (2013) Complete mitochondrial genome of the medicinal mushroom Ganoderma lucidum. PLoS ONE 8:e72038

Lowe TM, Eddy SR (1997) tRNAscan-SE: a program for improved detection of transfer RNA genes in genomic sequence. Nucl Acids Res 25:955-964

Ma H, Hagen F, Stekel DJ et al (2009) The fatal fungal outbreak on Vancouver Island is characterized by enhanced intracellular parasitism driven by mitochondrial regulation. Proc Natl Acad Sci USA 106:12980-12985

Monteiro-Vitorello CB, Bell JA, Fulbright DW, Bertrand H (1995) A cytoplasmically transmissible hypovirulence phenotype associated with mitochondrial DNA mutations in the chestnut blight fungus Cryphonectria parasitica. Proc Natl Acad Sci USA 92:5935-5939

Monteiro-Vitorello C, Hausner G, Searles D et al (2009) The Cryphonectria parasitica mitochondrial RNS gene: plasmid-like elements, introns and homing endonucleases. Fungal Genet Biol 46:837-848

Nakai R, Sen K, Kurosawa S, Shibai H (2000) Basidiomycetous fungus Flammulina velutipes harbors two linear mitochondrial plasmids encoding DNA and RNA polymerases. FEMS Microbiol Lett 190:99-102

Olson A, Stenlid J (2001) Plant pathogens: mitochondrial control of fungal hybrid virulence. Nature 411:438

Olson A, Aerts A, Asiegbu F et al (2012) Insight into trade-off between wood decay and parasitism from the genome of a fungal forest pathogen. New Phytol 194:1001-1013

Otrosina W, Garbelotto M (2010) Heterobasidion occidentale $\mathrm{sp}$ nov and Heterobasidion irregulare nom. nov.: a disposition of 
North American Heterobasidion biological species. Fungal Biol 114:16-25

Paquin B, Laforest MJ, Forget L et al (1997) The fungal mitochondrial genome project: evolution of fungal mitochondrial genomes and their gene expression. Curr Genet 31:380-395

Peden J (1999) Analysis of codon usage. University of Nottingham

Rogers HJ, Buck KW, Brasier CM (1987) A mitochondrial target for double-stranded RNA in diseased isolates of the fungus that causes Dutch elm disease. Nature 329:558-560

Rutherford K, Parkhill J, Crook J et al (2000) Artemis: sequence visualization and annotation. Bioinformatics 16:944-945

Stone C, Buitrago M, Boore J, Frederick R (2010) Analysis of the complete mitochondrial genome sequences of the soybean rust pathogens Phakopsora pachyrhizi and P. meibomiae. Mycologia 102:887-897

Torriani SFF, Penselin D, Knogge W et al (2014) Comparative analysis of mitochondrial genomes from closely related Rhynchosporium species reveals extensive intron invasion. Fungal Genet Biol 62:34-42
Touchon M, Nicolay S, Audit B et al (2005) Replication-associated strand asymmetries in mammalian genomes: toward detection of replication origins. Proc Natl Acad Sci USA 102:9836-9841

Wang Y, Zeng F, Hon CC et al (2008) The mitochondrial genome of the Basidiomycete fungus Pleurotus ostreatus (oyster mushroom). FEMS Microbiol Lett 280:34-41

Yoon H, You Y-H, Woo J-R et al (2012) The mitochondrial genome of the white-rot fungus Flammulina velutipes. J Gen Appl Microbiol 58:331-337

Yui Y, Katayose Y, Shishido K (1988) 2 linear plasmid-like DNA elements simultaneously maintained in Pleurotus Ostreatus. Biochim Biophys Acta 951:53-60

Zhao XQ, Aizawa T, Schneider J et al (2013) Complete mitochondrial genome of the aluminum-tolerant fungus Rhodotorula taiwanensis RS1 and comparative analysis of Basidiomycota mitochondrial genomes. Microbiol Open 2:308-317 